\section{Epidemiología de las muertes prematuras en Chile en la década 2001-2010}

\author{
ROBERTO BÄCHLER ${ }^{1}$, GLORIA ICAZA ${ }^{2, \mathrm{a}}$, ALEX SOTO ${ }^{2, \mathrm{~b}}$, \\ LORETO NÚÑEZ ${ }^{3, c}$, CAROLINA ORELLANA ${ }^{1, \mathrm{~d}}$, \\ ROLANDO MONSALVE', ${ }^{3, e}$ MARCELA RIQUELME ${ }^{3, e}$
}

\section{Epidemiology of premature deaths in Chile between 2001 and 2010}

Background: Years of potential life lost (YPLL) is a parameter that allows to analyze premature deaths. Aim: To study the causes, differences by gender, geographic variations and the trend of premature deaths in the last decade in Chile. Material and Methods: Using death databases published by the Ministry of Health, YPLL were calculated for the decade 2001-2010, using the method proposed by the Organization for Economic Cooperation and Development. Results: The standardized annual average YPLL rate in Chile in the study period was 4.703 per 100,000 inhabitants. Trauma, cancer and cardiovascular diseases accounted for $68 \%$ of YPLL. The male/female YPLL ratio was 2.07; for trauma and mental disorders, the male/female ratio for YPLL was over 5; for cardiovascular and respiratory diseases the male/female ratio was over two. Regions with a higher proportion of rural areas had higher YPLL rates. Conclusions: The main causes of YPLL are potentially preventable. Further studies should be carried out to identify risk factors for premature death.

(Rev Med Chile 2017; 145: 319-326)

Key words: Epidemiology; Life Expectancy; Mortality, premature.

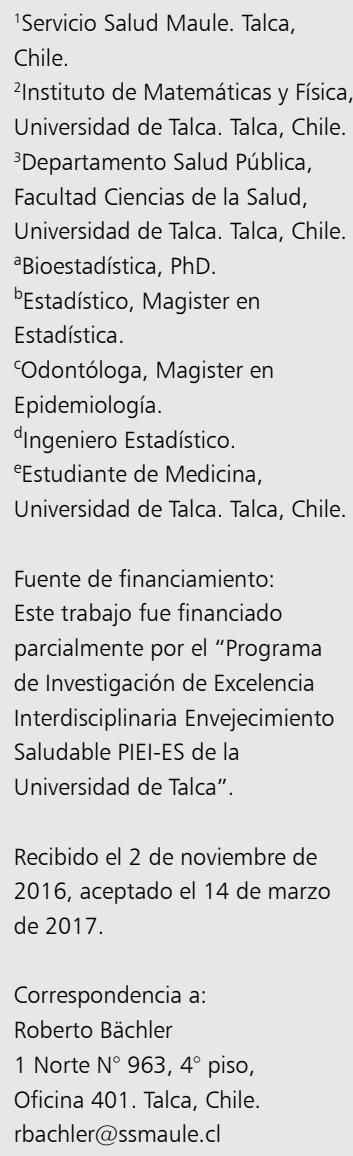

E l aumento de la expectativa de vida observado en las últimas décadas en los países que integran la Organización para la Cooperación y Desarrollo Económico (OECD), se asocia a un envejecimiento de la estructura demográfica de esos estados. El incremento de la cantidad de adultos mayores que se produce como consecuencia del fenómeno antes descrito, determina a su vez, un aumento de la tasa de mortalidad general en esas naciones.

El hecho que en estos países el riesgo poblacional de fallecer se incremente producto de la existencia de un mayor número de personas en edad avanzada, hace que la tasa bruta de mortalidad general vaya perdiendo relevancia como indicador para evaluar el daño en salud, y consecuentemente, su contribución a la definición de políticas públicas se hace cada vez menos significativa, toda vez que la muerte constituye un hecho natural al final del curso de la vida. Frente a esta realidad, surge la necesidad de identificar una variable que permita cuantificar de manera resumida las causas que ocasionan la muerte prematura de las personas, y a la vez, sirva para evaluar el resultado de las intervenciones y/o programas de salud dirigidos a los diferentes grupos poblacionales.

Una alternativa válida que da respuesta al problema mencionado en el párrafo precedente, está dada por el indicador Años de Vida Potencialmente Perdidos (AVPP). Esta variable se caracteriza por resumir en una sola cifra, los años que la sociedad pierde como consecuencia de la 
muerte prematura de las personas, el concepto cuantificado a través de esta medición, constituye una herramienta epidemiológica que aporta valiosa información, respecto de las causas que ocasionan el fallecimiento de personas en etapas tempranas de la vida; así mismo, representa un instrumento eficaz para evaluar el impacto de las políticas públicas en el ámbito de la salud.

Las publicaciones de los organismos internacionales que han estudiado el tema de las muertes prematuras utilizando como unidad de análisis los AVPP difundidas en las últimas décadas, muestran que el riesgo global de fallecer en edades tempranas de la vida ha disminuido considerablemente en los países que presentan una mejoría en su desarrollo socio económico; específicamente, el documento publicado por la OECD "Health at Glance 2011", muestra que la tasa de AVPP de los países que integran dicho organismo, disminuyó de 8.727 por 100.000 habitantes el año 1970 a 3.544 por 100.000 habitantes el año $2009^{1}$.

Chile, es un país que no ha estado ajeno a las tendencias antes descritas, de hecho la expectativa de vida de sus habitantes ha experimentado un incremento de más de 20 años en las últimas cinco décadas ${ }^{1}$, ello ha determinado que su estructura demográfica, al igual que la de los países desarrollados, comience a envejecer. En este contexto, se ha estimado procedente investigar el fenómeno de las muertes prematuras en un país en vías de desarrollo. El objetivo de este estudio es conocer la realidad epidemiológica de las muertes prematuras en Chile, y a la vez, aportar información válida que contribuya a la definición de políticas públicas respecto de aquellos problemas que están determinando fallecimientos en etapas tempranas de la vida.

\section{Material y Métodos}

Se calculó los AVPP registrados en Chile en el decenio 2001-2010, para ello se utilizó las bases de datos de defunciones del Departamento de Estadística e Información en Salud del Ministerio de Salud de Chile $^{2}$, para analizar la variable objeto de estudio (AVPP) se estableció como límite inferior un año de edad y como límite superior setenta años, las tasas fueron estandarizadas utilizando la metodología definida por la OECD ${ }^{3,4}$. La investigación consideró estudiar las causas que producen las muertes prematuras y un análisis por sexo, por área geográfica y la tendencia del decenio. Adicionalmente, se ajustó un modelo lineal para la tasa estandarizada de AVPP en función del tiempo, las áreas geográficas (regiones) y la interacción entre ambas variables, para ello se asume un efecto lineal de los AVPP en el decenio estudiado; a partir de este diseño se realizó un estudio de conglomerados (cluster) utilizando la media, la desviación estándar y la pendiente del modelo lineal, como resultado de este último análisis se obtuvieron tres conglomerados. Para cada uno de los conglomerados identificados, se construyeron modelos lineales y se compararon las diferencias de tendencia entre ellos. El estudio de las causas de muerte se realizó usando la Clasificación Internacional de Enfermedades en su décima versión -CIE $10{ }^{5}$. El análisis estadístico se hizo utilizando el software SPSS versión 15.0 y SAS versión 9.3, para la presentación de los resultados se usaron estadígrafos convencionalmente aceptados en la literatura epidemiológica. En la Figura 1 se presenta la expresión matemática utilizada para calcular los AVPP.

\section{Resultados}

\section{Análisis por causa}

Chile registró un promedio anual de 623.556 AVPP en el decenio 2001-2010, esto determinó una tasa anual estandarizada promedio de 4.703,10 AVPP por 100.000 habitantes. Las principales causas de años de vida perdidos como consecuencia de una muerte prematura correspondieron a traumatismos, neoplasias (tumores malignos) y enfermedades del aparato circulatorio, estas tres causas en su conjunto representan

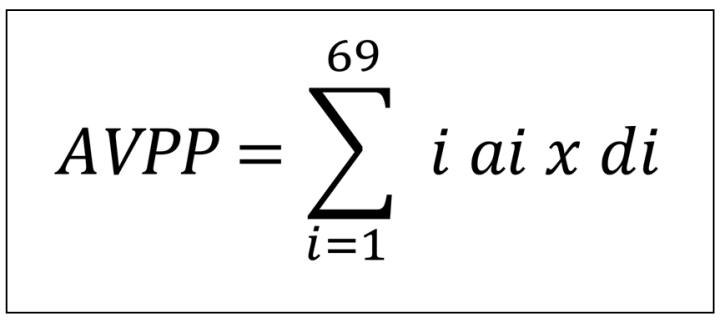

Figura 1. $a=$ Diferencia de años de vida hasta la edad de 70 años, cuando la defunción ocurre entre la edad i e i + 1; $\mathrm{d}=$ número de muertes entre la edad i e $\mathrm{i}+1$. 
68,03\% de los AVPP registrados en el país en el período en estudio. Las enfermedades del aparato digestivo, las infecciosas y parasitarias, las del sistema respiratorio, las del sistema nervioso junto con las endocrinas, nutricionales y metabólicas representan 23,10\% de los AVPP registrados en el decenio analizado. Los AVPP originados por otras causas constituyen una proporción marginal respecto a los grupos antes mencionados (Tabla 1).

\section{Análisis por sexo}

El análisis por sexo muestra que la razón hombre/mujer de la tasa anual estandarizada promedio de AVPP en el decenio estudiado es 2,07; en aquellos casos en que la causa de muerte fue un traumatismo, un trastorno mental y del comportamiento esta razón es superior a 5. En el caso de las enfermedades del aparato digestivo y las infecciosas y parasitarias, la tasa anual promedio de los hombres fue casi el triple que la de las mujeres. Para las enfermedades del sistema circulatorio y respiratorio la tasa promedio anual de los hombres es el doble que las mujeres. En aquellos casos que la causa de muerte prematura fue una neoplasia la razón hombre/mujer no muestra diferencias (Tabla 2).

\section{Análisis geográfico}

El análisis de la tasa estandarizada de los AVPP de las quince regiones de Chile muestra importan-

Tabla 1. Causas de años de vida potencialmente perdidos Chile 2001-2010

\begin{tabular}{|c|c|c|c|c|}
\hline & Capítulos C.I.E 10 & $\begin{array}{c}\text { AVPP anual } \\
\text { promedio } \\
2001-2010\end{array}$ & $\begin{array}{c}\text { Tasa anual } \\
\text { estandarizada } \\
\text { promedio }^{1}\end{array}$ & $\%$ \\
\hline XIX & $\begin{array}{l}\text { Traumatismos, envenenamientos y algunas otras consecuencias } \\
\text { de causa externa }\end{array}$ & 203.967 & $1.316,09$ & 32,71 \\
\hline$\|$ & Neoplasias & 132.448 & $1.103,72$ & 21,24 \\
\hline IX & Enfermedades del sistema circulatorio & 87.820 & 761,71 & 14,08 \\
\hline$X I$ & Enfermedades del aparato digestivo & 53.740 & 445,78 & 8,62 \\
\hline $\mathrm{VI}$ & Enfermedades del sistema nervioso & 25.571 & 165,73 & 4,10 \\
\hline$x$ & Enfermedades del sistema respiratorio & 24.201 & 195,27 & 3,88 \\
\hline I & Ciertas enfermedades infecciosas y parasitarias & 23.619 & 168,85 & 3,79 \\
\hline IV & Enfermedades endocrinas, nutricionales y metabólicas & 16.942 & 146,94 & 2,72 \\
\hline $\mathrm{XVIII}$ & $\begin{array}{l}\text { Síntomas, signos y hallazgos anormales clínicos y de laboratorio, } \\
\text { no clasificados en otra parte }\end{array}$ & 12.599 & 89,36 & 2,02 \\
\hline$X V I I$ & $\begin{array}{l}\text { Malformaciones congénitas, deformidades y anomalías cromo- } \\
\text { sómicas }\end{array}$ & 10.348 & 59,72 & 1,66 \\
\hline III & $\begin{array}{l}\text { Enfermedades de la sangre y de los órganos hematopoyéticos } \\
\text { y otros trastornos que afectan el mecanismo de la inmunidad }\end{array}$ & 9.583 & 70,69 & 1,54 \\
\hline V & Trastornos mentales y del comportamiento & 7.666 & 60,26 & 1,23 \\
\hline XIV & Enfermedades del aparato genitourinario & 7.605 & 64,97 & 1,22 \\
\hline XIII & Enfermedades del sistema osteomuscular y del tejido conectivo & 4.710 & 35,22 & 0,76 \\
\hline $\mathrm{XV}$ & Embarazo, parto y puerperio & 1.701 & 20,99 & 0,27 \\
\hline XII & Enfermedades de la piel y el tejido subcutáneo & 578 & 4,86 & 0,09 \\
\hline$X V I$ & Ciertas afecciones originadas en el período perinatal & 363 & 2,17 & 0,06 \\
\hline \multirow[t]{2}{*}{ VIII } & Enfermedades del oído y de la apófisis mastoides & 94 & 1,18 & 0,02 \\
\hline & Total general & 623.556 & $4.703,10$ & 100 \\
\hline
\end{tabular}

${ }^{1}$ Tasa por 100.000 habitantes. 
Tabla 2. Causas de años de vida potencialmente perdidos según sexo Chile 2001-2010

\begin{tabular}{|c|c|c|c|c|}
\hline & Capítulos C.I.E 10 & \multicolumn{2}{|c|}{ Tasa anual promedio' } & $\begin{array}{c}\text { Razón } \\
\text { Hombre/Mujer }\end{array}$ \\
\hline XIX & $\begin{array}{l}\text { Traumatismos, envenenamientos y algunas otras consecuencias } \\
\text { de causa externa }\end{array}$ & $2.209,47$ & 440,67 & 5,01 \\
\hline ॥ & Neoplasias & $1.107,76$ & $1.108,19$ & 1,00 \\
\hline IX & Enfermedades del sistema circulatorio & $1.037,51$ & 507,49 & 2,04 \\
\hline$X I$ & Enfermedades del aparato digestivo & 679,94 & 227,09 & 2,99 \\
\hline$x$ & Enfermedades del sistema respiratorio & 264,70 & 129,94 & 2,04 \\
\hline । & Ciertas enfermedades infecciosas y parasitarias & 254,99 & 85,36 & 2,99 \\
\hline VI & Enfermedades del sistema nervioso & 202,15 & 130,41 & 1,55 \\
\hline IV & Enfermedades endocrinas, nutricionales y metabólicas & 165,55 & 129,55 & 1,28 \\
\hline XVIII & $\begin{array}{l}\text { Síntomas, signos y hallazgos anormales clínicos y de laborato- } \\
\text { rio, no clasificados en otra parte }\end{array}$ & 126,30 & 54,33 & 2,32 \\
\hline $\mathrm{V}$ & Trastornos mentales y del comportamiento & 104,29 & 18,16 & 5,74 \\
\hline III & $\begin{array}{l}\text { Enfermedades de la sangre y de los órganos hematopoyéticos } \\
\text { y otros trastornos que afectan el mecanismo de la inmunidad }\end{array}$ & 80,02 & 61,92 & 1,29 \\
\hline XIV & Enfermedades del aparato genitourinario & 69,65 & 60,89 & 1,14 \\
\hline XVII & $\begin{array}{l}\text { Malformaciones congénitas, deformidades y anomalías } \\
\text { cromosómicas }\end{array}$ & 60,26 & 59,48 & 1,01 \\
\hline XIII & Enfermedades del sistema osteomuscular y del tejido conectivo & 19,90 & 50,44 & 0,39 \\
\hline XII & Enfermedades de la piel y el tejido subcutáneo & 6,03 & 3,79 & 1,59 \\
\hline$X \mathrm{VI}$ & Ciertas afecciones originadas en el período perinatal & 2,50 & 2,44 & 1,02 \\
\hline VIII & Enfermedades del oído y de la apófisis mastoides & 1,07 & 0,40 & 2,65 \\
\hline \multirow[t]{2}{*}{ XV } & Embarazo, parto y puerperio & 0 & 21,20 & 0 \\
\hline & Total general & $6.392,08$ & $3.091,74$ & 2,07 \\
\hline
\end{tabular}

${ }^{1}$ Tasa por 100.000 habitantes.

tes variaciones geográficas. En términos generales se puede señalar que las regiones de Los lagos, Aysén, Los Ríos y Magallanes son las que presentan las mayores tasas en el período en estudio. En una situación intermedia, con cifras cercanas a la mediana nacional se observan las regiones de Tarapacá, Antofagasta, O’Higgins y Maule. En el otro extremo las regiones con las menores tasas son Coquimbo, Atacama y la Región Metropolitana (Figura 2).

\section{Análisis de conglomerados}

El análisis de conglomerados identificó tres grupos, el grupo 1 considera las regiones de Tarapacá, Valparaíso, Región Metropolitana, O’Higgins, Maule, Los Ríos y Magallanes; el grupo
2 incorpora las regiones de Biobío, Araucanía y Aisén; por su parte el grupo 3 incluye las regiones de Arica y Parinacota, Antofagasta, Atacama, Coquimbo y Los Lagos. El grupo 2 presenta la mayor tasa media de AVPP, por su parte en el grupo 3 se registra la menor tasa de muertes prematuras en el período en estudio. En la Figura 3 se presenta la tendencia de los conglomerados en el decenio y en la Tabla 3 se resume los principales parámetros estadísticos de cada grupo.

\section{Discusión}

La primera causa de AVPP en Chile en el período 2001-2010 fueron los traumatismos, en- 

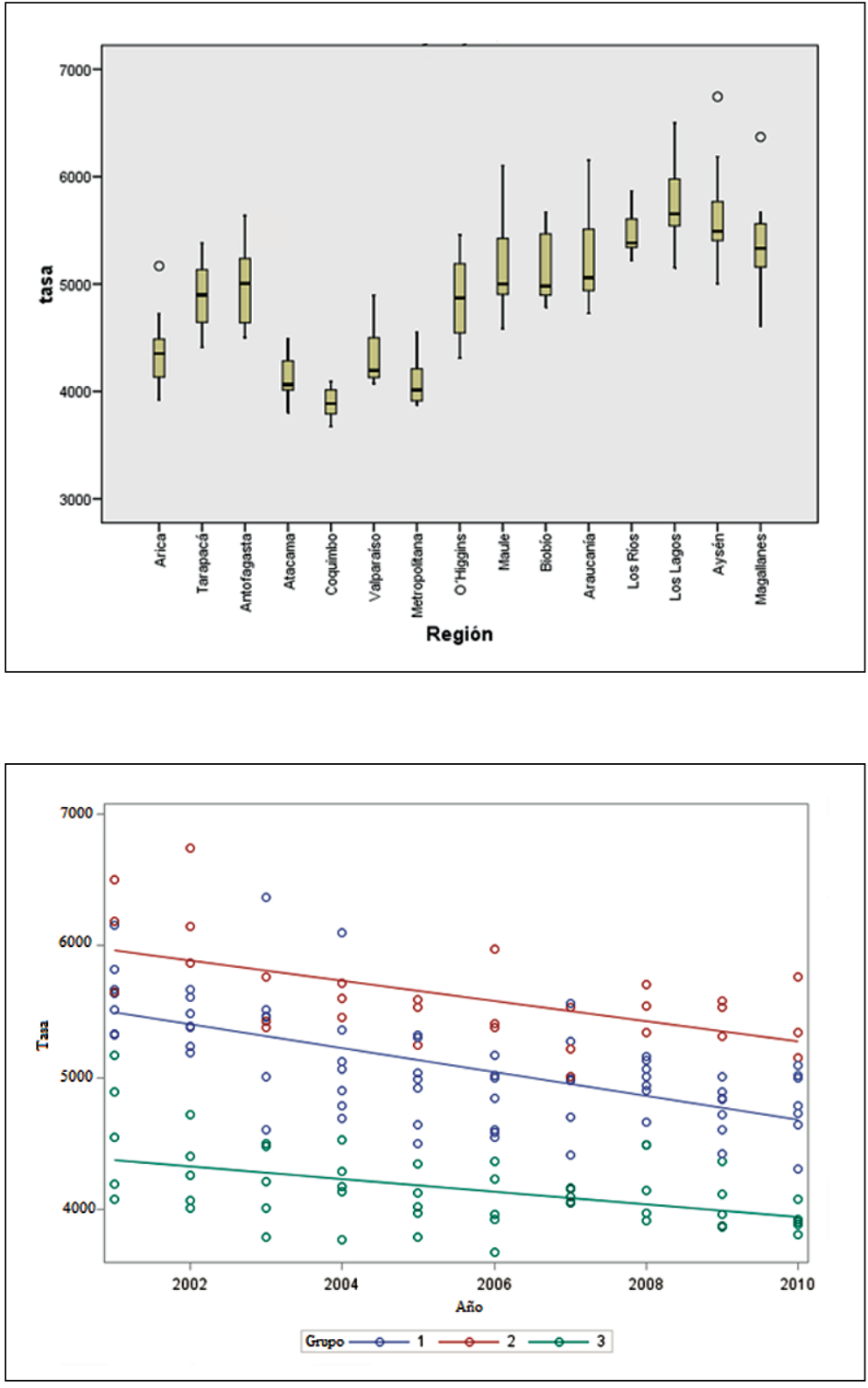

Figura 2. Años de vida potencialmente perdidos por regiones de Chile, Chile 2001-2010. Tasa estandarizada por 100.000 habitantes.

Figura 3. Años de vida potencialmente perdidos conglomerados, Chile 20012010.

Tabla 3. Parámetros modelo lineal conglomerados AVPP 2001-2010, Chile

\begin{tabular}{|cccc|}
\hline Grupo & $\begin{array}{c}\text { Pendiente } \\
(\mathbf{m})\end{array}$ & $\begin{array}{c}\text { Error } \\
\text { estándar }\end{array}$ & p \\
\hline 1 & $-0,90427$ & 0,13738 & $<0,001$ \\
\hline 2 & $-0,75867$ & 0,20291 & 0,0008 \\
\hline 3 & $-0,48058$ & 0,13039 & 0,0006 \\
\hline
\end{tabular}

venenamientos y algunas otras consecuencias de causa externa, este problema de salud representa $1 / 3$ de los AVPP registrados en Chile en el decenio estudiado. Si bien, en el mundo se ha registrado una considerable disminución de las muertes prematuras en los últimos decenios ${ }^{1}$, en el caso particular de Estados Unidos en el período 20102014, se ha observado que el número de muertes prevenibles por traumatismos no intencionales se 
ha incrementado ${ }^{6}$; este hecho debe ser motivo de preocupación y estudio, toda vez que las muertes por este problema de salud corresponden a defunciones no naturales susceptibles de prevenir; el desafío entonces es identificar las causas que originan este tipo de defunciones prematuras, $\mathrm{y}$ a partir de esos antecedentes, elaborar políticas y/o programas preventivos orientados a disminuir este daño.

El análisis de AVPP por sexo realizado en la presente investigación muestra que la razón hombre/mujer es 2,07, este hallazgo es coincidente con lo señalado en la literatura científica, las publicaciones relativas a esta materia muestran sistemáticamente que el riesgo de muerte prematura es mayor en los hombres que en las mujeres ${ }^{7-24}$. En el caso particular de los trastornos mentales y del comportamiento y, de los traumatismos, envenenamientos y algunas otras causas externas la razón hombre/mujer es mayor de 5. Sólo en las enfermedades del sistema osteomuscular y del tejido conectivo los AVPP en las mujeres son mayores que lo observado en los hombres, en estas últimas patologías la razón hombre/mujer es 0,39 . El mayor riesgo de muertes prematuras de los hombres respecto a las mujeres representa una interesante área de investigación epidemiológica; el desarrollo de estudios orientados a la identificación de factores de riesgo por causas específicas de muerte prematura según sexo, permitirá disponer de mayor evidencia científica respecto a este fenómeno.

En lo que dice relación con el análisis geográfico se puede señalar que las diferencias observadas en las tasas de AVPP entre las distintas regiones, pueden ser explicadas entre otras razones, por la mayor o menor accesibilidad que tienen las personas a los centros de salud desde su residencia, esta afirmación se fundamenta en el hecho que en las regiones de Chile que tienen una mayor ruralidad, como lo son, Los Lagos, Aysén y Los Ríos, se registra una mayor tasa de muertes prematuras respecto a las regiones donde la población vive preferentemente en conglomerados urbanos, como lo son la Región Metropolitana y de Coquimbo. Un análisis de causas especificas de muerte por regiones, entregaría elementos analíticos complementarios relativos a la influencia que tendría, por ejemplo, la accesibilidad a atención médica en centros de alta complejidad, y la frecuencia de muertes prematuras por politraumatismos y de infarto al miocardio, patologías ambas, para las cuales está demostrado el beneficio de una atención oportuna en unidades de cuidados intensivos ${ }^{25-27}$.

El modelo lineal del análisis por conglomerados, si bien muestra que en los tres grupos la tendencia de los AVPP en el decenio fue a la disminución, existe diferencias en el nivel de la tasa como en la pendiente de la misma. El grupo 2, que incluye a regiones con una alta ruralidad (Biobío, Araucanía y Aisén) es el que presenta la mayor tasa de muertes prematuras con una pendiente de descenso menor que el grupo que aparece en un nivel intermedio (grupo 1), por su parte el grupo 3 es el que presenta la menor tasa de AVPP y en la cual la pendiente de la tendencia es menor, estas diferencias se explicarían, entre otras razones, porque en este último grupo están incluidas las regiones donde la gente vive preferentemente en conglomerados urbanos.

Otras variables relevantes a considerar en estudios de muertes prematuras no incluidas en esta investigación debido a que actualmente no se registran en los certificados de defunción, dicen relación la presencia de factores de riesgo asociados a enfermedades crónicas no transmisibles como: obesidad, sedentarismo, tabaquismo, consumo exagerado de alcohol, hipertensión arterial no controlada e hiperlipidemia, la evidencia respecto a esta materia demuestra que las personas que no presentan estos factores tienen un menor riesgo de muerte prematura ${ }^{28}$. Para disminuir el riesgo de muertes prematuras ocasionadas por este grupo de enfermedades, el Centro para el Control y Prevención de Enfermedades (CDC) de Estados Unidos ha propuesto estrategias específicas para los ámbitos gubernamentales y comunitarios, las cuales deben complementarse con intervenciones poblacionales que promuevan estilos de vida saludables y garanticen el adecuado control de las personas que presentan enfermedades crónicas no transmisibles ${ }^{29}$.

Adicionalmente, es necesario señalar que los estudios de muertes prematuras a través del parámetro AVPP, al igual que cualquier análisis de mortalidad, se realizan sobre la base de los registros disponibles de fallecidos, los cuales habitualmente corresponden a los certificados de defunción. La validez de estas investigaciones está determinada por la integridad del registro, esto es que incluya todos los casos del fenómeno en estudio, en este caso las muertes, y por la cali- 
dad de los datos consignados en el certificado de defunción. En el caso de Chile, la integridad del registro es prácticamente $100 \%$ y los certificados de defunción son emitidos básicamente por profesionales médicos, estas particularidades permiten garantizar la validez de los resultados obtenidos en la presente investigación.

El conocimiento generado a partir de la presente investigación, además de entregar información epidemiológica respecto al problema de las muertes prematuras en Chile, permite identificar los grandes grupos de causas en los cuales sería recomendable implementar y/o reforzar programas preventivos. Un trabajo sistematizado en esta área, constituye la base para seguir avanzando en mejorar el nivel de salud de la población.

\section{Referencias}

1. Health at a Glance 2011, OECD Indicators. Publicación disponible en: http://dx.doi.org/10.1787/health_glance2011-en.

2. Departamento de Estadísticas e Información de Salud, Ministerio de Salud, Chile. Disponible en: http://www. deis.cl/

3. Conti S, Masocco M, Farchi G, Rezza G, Toccaceli V. Premature mortality in Italy during the first decade of the AIDS epidemic: 1984-1993. Int J Epidemiol 1997; 26 (4): 873-9.

4. OECD Health Data 2012. Definitions, Sources and Methods. Disponible en: http://www.oecd.org/health/ healthdat.

5. International Statistical Classification of Diseases and Related Health Problems 10th Revision. Disponible en http://apps.who.int/classifications/icd10/browse/2016/ en.

6. García MC, Bastian B, Rossen LM, Anderson R, Miniño A, Yoon PW, et al. Potentially Preventable Deaths Among the Five Leading Causes of Death-United States, 2010 and 2014. MMWR Morb Mortal Wkly Rep. 2016; 65 (45): 1245-55.

7. Arcà M, di Orio F, Forastiere F, Tasco C, Perucci CA. Years of potential life lost (YPLL) before Age 65 in Italy. Am J Public Health 1988; 78 (9): 1202-5.

8. McDonnell S, Vossberg K, Hopkins RS, Mittan B. Using YPLL in Health Planning. Public Health Rep 1998; 113 (1): 55-61.

9. O'Shea E. Social gradients in years of potential life lost in Ireland. Eur J Public Health 2003; 13 (4): 327-33.

10. Mariotti S, D'Errigo P, Mastroeni S, Freeman K. Years of life lost due to premature mortality in Italy. Eur J Epidemiol 2003; 18 (6): 513-21.

11. Artnik B, Vidmar G, Javornik G, Laaser U. Premature Mortality in Slovenia in relation to selected biological, socioeconomic, and geographical determinants. Croat Med J 2006; 47 (1): 103-13.

12. Génova-Maleras R, Catalá-López F, de Larrea-Baz N, Álvarez-Martín E, Morant-Ginestar C. The burden of premature mortality in Spain using standard expected years of life lost: a population-based study. BMC Public Health 2011; 11: 787.

13. Poorolajal J, Esmailnasab N, Ahmadzadeh J, Motlagh TA. The Burden of Premature Mortality in Hamadan Province in 2006 and 2010 Using Standard Expected Years of Potential Life Lost: A Population-based Study. Epidemiol Health 2012; 34: e2012005.

14. Plass D, Chau PY, Thach TQ, Jahn HJ, Lai PC, Wong $\mathrm{CM}$, et al. Quantifying the burden of disease due to premature mortality in Hong Kong using standard expected years of life lost. BMC Public Health 2013; 13: 863.

15. Rennert G. Premature mortality, Israel 1986. Isr J Med Sci 1992; 28 (7): 442-6.

16. Ramírez de Arellano AB. The death divide: differentials in premature mortality by gender in Puerto Rico. Bol Asoc Med P R 1992; 84: 11-4.

17. Humblet PC, Lagasse R, Leveque A. Trends in Belgian premature avoidable deaths over a 20 year period. J Epidemiol Community Health 2000; 54 (9): 687-91.

18. Semerl JS, Sesok J. Years of potential life lost and valued years of potential life lost in assessing premature mortality in Slovenia. Croat Med J. 2002; 43: 439-45.

19. Naci H, Baker TD. Productivity losses from road traffic deaths in Turkey. Int J Inj Contr Saf Promot 2008; 15 (1): 19-24.

20. Vlajinac H, Marinkovic J, Kocev N, Sipetic S, Bjegovic $\mathrm{V}$, Jankovic S, et al. Years of life lost due to premature death in Serbia (excluding Kosovo and Metohia). Public Health 2008; 122 (3): 277-84.

21. Santric Milicevic M, Bjegovic V, Terzic Z, Vukovic D, Kocev N, Marinkovic J, et al. Serbia within the European context: An analysis of premature mortality. Popul Health Metr 2009; 7: 12.

22. Kim KS, Kim SD, Lee SH. Trend of mortality rate and injury burden of transport accidents, suicides, and falls. J Prev Med Public Health 2012; 45 (1): 8-13.

23. Lukaschek K, Erazo N, Baumert J, Ladwig KH. Suicide mortality in comparison to traffic accidents and homicides as causes of unnatural death. An analysis of 14,441 cases in Germany in the year 2010. Int J Environ Res Public Health 2012; 9 (3): 924-31. 
24. Leyland A. H. Increasing inequalities in premature mortality in Great. Britain. J Epidemiol Community Health 2004; 58 (4): 296-302.

25. ESC Guidelines for the management of acute myocardial infarction in patients presenting with ST-segment elevation. The Task Force on the management of ST-segment elevation acute myocardial infarction of the European Society of Cardiology (ESC). European Heart Journal 2012; 33: 2569-619.

26. Corbalán R, Nazzal C, Prieto JC, Chávez E, Lanas F, Lamich $\mathrm{R}$, et al. Reducción de la mortalidad por infarto del miocardio en hospitales chilenos. Rev Med Chile 2002; 130 (4): $368-78$
27. National Institute for Health and care Excellence. Major trauma: service delivery NICE guideline, 2016, disponible en https://www.nice.org.uk/guidance/ng40/

28. Veronese N, Li Y, Manson JE, Willett WC, Fontana $\mathrm{L}, \mathrm{Hu}$ FB. Combined associations of body weight and lifestyle factors with all cause and cause specific mortality in men and women: prospective cohort study. BMJ 2016; 355: 5855.

29. Bauer UE, Briss PA, Goodman RA, Bowman BA. Prevention of chronic disease in the 21st century: elimination of the leading preventable causes of premature death and disability in the USA. Lancet 2014; 384 : 45-52. 